\section{Article}

Cite this article: Altena B, Kääb A (2020). Ensemble matching of repeat satellite images applied to measure fast-changing ice flow, verified with mountain climber trajectories on Khumbu icefall, Mount Everest. Journal of Glaciology 66(260), 905-915. https://doi.org/ 10.1017/jog.2020.66

Received: 3 February 2020

Revised: 14 July 2020

Accepted: 15 July 2020

First published online: 11 August 2020

\section{Keywords:}

Glacier flow; ice velocity; remote sensing

Author for correspondence:

Bas Altena,

E-mail: bas.altena@geo.uio.no

\title{
Ensemble matching of repeat satellite images applied to measure fast-changing ice flow, verified with mountain climber trajectories on Khumbu icefall, Mount Everest
}

\section{Bas Altena ${ }^{1,2}(\mathbb{D})$ and Andreas Kääb ${ }^{1}$ (D)}

${ }^{1}$ Department of Geosciences, University of Oslo, Oslo, Norway and ${ }^{2}$ Institute for Marine and Atmospheric Research, Utrecht University, Utrecht, The Netherlands

\begin{abstract}
Velocities within an icefall are typically the fastest within a glacier system and experience complex flow. The combination of convergent and fast flow, and steep slope generate a quickly changing and intensely fractured surface. This complicates velocity extraction from repeat satellite images, especially when common pattern matching procedures are used. In this study, we exploit the high temporal revisit of medium-resolution satellite images using a novel image matching technique, ensemble matching, making it possible to generate a high-resolution $(30 \mathrm{~m})$ velocity field from high-repeat image sequences despite challenging image conditions. We demonstrate this technique for the first time in the glaciology domain using repeat Sentinel-2 optical data over the famous Khumbu icefall, situated on the southern slopes of Mount Everest. Estimates of velocity go just over $1 \mathrm{~m} \mathrm{~d}^{-1}$, which is slower than summer velocities from noisy single pair image matching. This icefall is frequently crossed by high-altitude mountaineers who use a route confined by fixed ropes and ladders set out every season. The mountain climbers typically record their trajectory on their personal satellite navigation device. We use such volunteered geographic information to verify our velocity estimates, confirming our underestimation with ensemble matching. Besides unprecedented remotely sensed surface velocities over the icefall, we also note that the generated velocity field can aid with the planning of a safe passage through this icefall.
\end{abstract}

\section{Introduction}

When ice flow on a glacier converges and bedrock steepens, an icefall can originate. Icefalls are highly fractured and are characterized by complicated or chaotic and fast-changing crevasse patterns. Though dangerous, the Khumbu icefall $\left(27.99^{\circ} \mathrm{N} 86.87^{\circ} \mathrm{E}\right)$ is the only possible passage when climbing Mount Everest on the normal route from the Nepali side. At this icefall, and likewise at many others, the glacier flows through a narrow passage, with confined and steep valley walls (Fig. 1) making it also prone to avalanches and rockfall. Leading to the highest peak on Earth makes the icefall a frequently visited destination and many climbers follow a route through the icefall that is predefined and installed every season. This route comes not without danger, as demonstrated by an avalanche in 2009 that killed three people (Moore and others, 2017) and later again in 2014, when 16 climbers died (Stokes and others, 2015). In both tragic events, all these casualties were local mountain workers, who were preparing the fixed ropes and aluminum ladders in the icefall. Not seldom in the Himalayas, local mountain guides spend a particularly long exposure period within dangerous sections of frequently used touristic climbing routes, making them more susceptible to deadly incidents (Firth and others, 2008). Hence, any risk reduction which can help these local mountain workers (also known as ice doctors) should be of great value. One of such measures is the mapping of velocity gradients, as crevasses originate when a threshold in shear strength and strain rate is surpassed (Colgan and others, 2016), which can be done from reliable velocity maps.

Apart from mapping shear zones for safety issues, information about the flow characteristics in icefalls is useful for a number of glaciological applications. For example, icefalls can be hydrological barriers between the accumulation region and the ablation area, as is suggested both from boreholes (Harper and Humphrey, 1995) and velocity evolution (Durkin and others, 2017; Armstrong and others, 2017). Also, percolation of water and advection of air into the glacier and to the bedrock is more easy though the intense fracturing at the surface (Jarvis and Clarke, 1974; Kodama and Mae, 1976), making icefalls zones of potentially increased interaction with atmospheric conditions and their changes (Gilbert and others, 2020).

Glacier behavior at icefalls is characterized by fast-changing and chaotic ice flow velocity. These are particularly difficult to measure under associated conditions. In situ measurements are almost impossible, except from (automatic) time-lapse cameras (Messerli and Grinsted, 2015; Giordan and others, 2016). Measurements based on repeat satellite imagery, which are well established for other zones of glaciers (Heid and Kääb, 2012; Dehecq and others, 2015), tend to fail over icefalls as the ice is flowing too fast, trackable surface patterns are changing too much, and the strong lateral velocity gradients deteriorate accurate window-based 


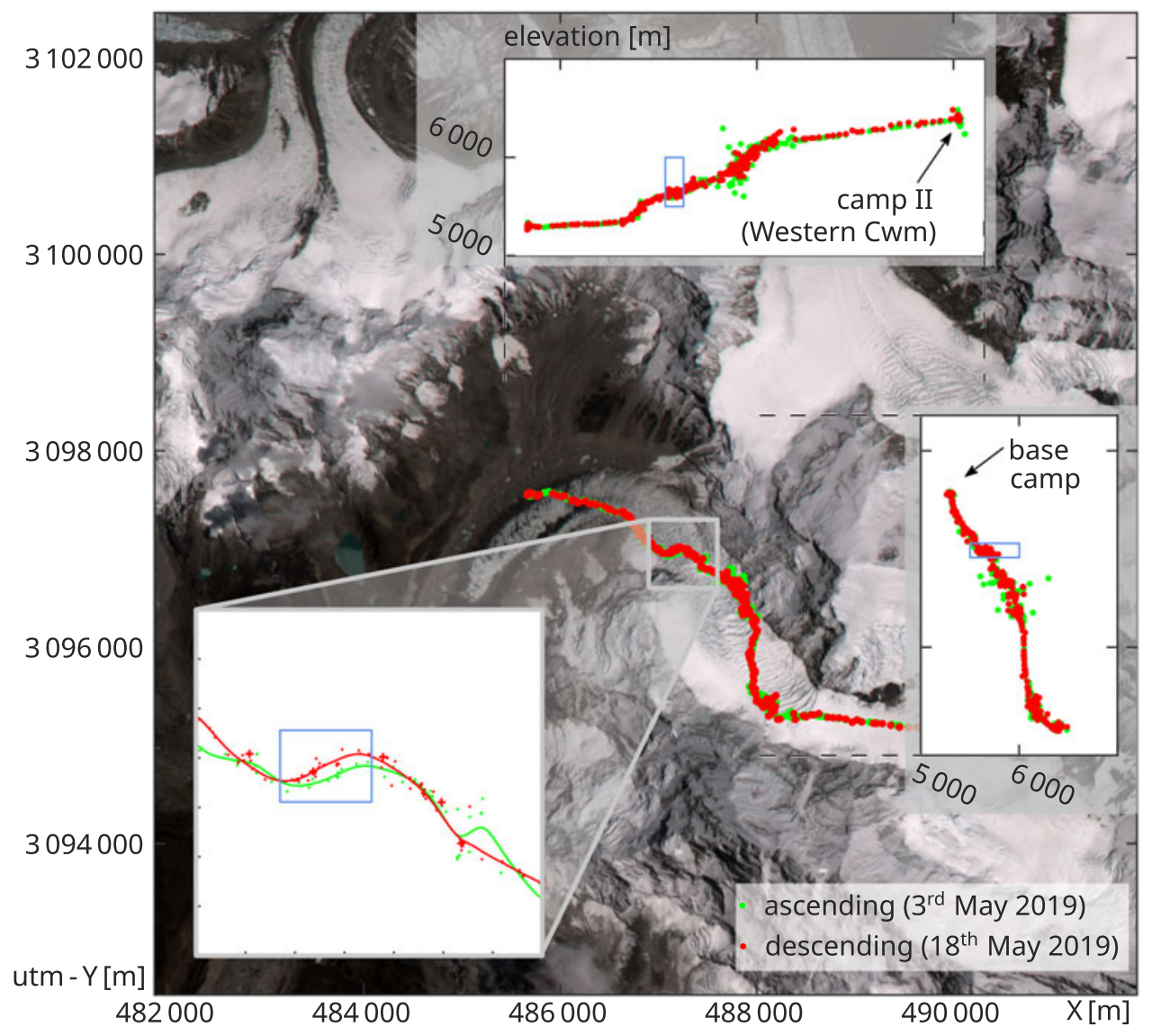

Fig. 1. Natural color imagery over Khumbu icefall in the Himalaya range. On the left side is the debris covered Khumbu Glacier, on the right side the top of Mount Everest and Lhotse are almost visible. The GPS points used in this study are overlain on this image. Only the trajectories from Everest base camp to Camp II are shown.

pattern matching. In this contribution, we use ensemble matching (also known as time-resolved particle image velocimetry), a technique novel to glaciology, and demonstrate the effectiveness of it using high-repeat Sentinel-2 optical satellite data of Khumbu icefall. The purpose of this contribution is a brief demonstration of the method for glaciologic application, rather than a in-depth technical study or detailed investigation of the ice flow in Khumbu icefall.

\section{Ensemble image matching}

\section{Displacement estimation from image matching in the spatial domain}

The foundation for displacement estimation from repeat remote sensing is pattern matching (Scambos and others, 1992; Kääb and Vollmer, 2000; Strozzi and others, 2002; Debella-Gilo and Kääb, 2011). It is based on finding a similar template from an image in another larger subsection of an image at a different time instance $\left(t_{q}\right)$. Often the similarity measure $(\rho)$ is implemented through normalized cross-correlation as,

$$
\rho_{p q}=\frac{\sum_{k, l}\left(\mathbf{I}_{p}(i+k, j+l)-\overline{\mathbf{I}}_{p}\right)\left(\mathbf{I}_{q}(k, l)-\overline{\mathbf{I}}_{q}\right)}{\sqrt{\sum_{k, l}\left(\mathbf{I}_{p}(i+k, j+l)-\overline{\mathbf{I}}_{p}\right)^{2} \sum_{k, l}\left(\mathbf{I}_{q}(k, l)-\overline{\mathbf{I}}_{q}\right)^{2}}} .
$$

Here, $\rho_{p q}$ denotes a score surface at discrete step sizes $(k, l)$. It is calculated by the similarity of two templates $\left(\mathbf{I}_{p}\right.$ and $\left.\mathbf{I}_{q}\right)$ at different time instances $\left(t_{p}\right.$ and $\left.t_{q}\right)$. The upper part of the fraction of equation (1) shifts the intensities in the templates by their mean intensity (denoted by the overbar) to transform the signals to zero-centered variations, while the lower part of the fraction normalizes the contrast of the templates (or intensity spread). This results in a similarity score that can range between -1 and +1 .

Matching the velocity over an icefall, or other similarly fast changing surfaces, is much more challenging than on a slow and homogenous flowing glacier tongue. Surface features in an icefall can change quickly over time, thus visual distinctive features, which can be tracked over time, are quickly lost. Furthermore, the flow is first converging and then diverging and thus exhibits considerable shear. Consequently, bulk velocity estimation (of homogenous, simple translation) does not (or not well) work, and in order to fulfill this constrain smaller template sizes are needed, which then lowers the signal-to-noise level (Debella-Gilo and Kääb, 2012). Therefore, in this specific case, we apply pattern matching in the time domain (Meinhart and others, 2000), known as ensemble matching (Delnoij and others, 1999), see also the schematic in Figure 2. This means the matching is done with much smaller windows than necessary for common single image pair matching, but instead information is gathered from multiple image pairs of a whole collection. Because some of the imagery will have cloud cover, not all image pairs can be used from the stack, and therefore a selection is made and this we call a collection. With ensemble matching, the same or higher signal-to-noise can be achieved, as a similar total number of image samples (pixels) is taken as is done with single image pair matching, i.e. replacing spatial redundancy by temporal redundancy. When the flow does not change over the analyzed collection period, a clear peak will emerge. Laboratory experiments of sparsely illuminated seeds within a fluid or airmass have shown this spatial resolution can be reduced to a single pixel resolution of independent flow estimates, when an image 

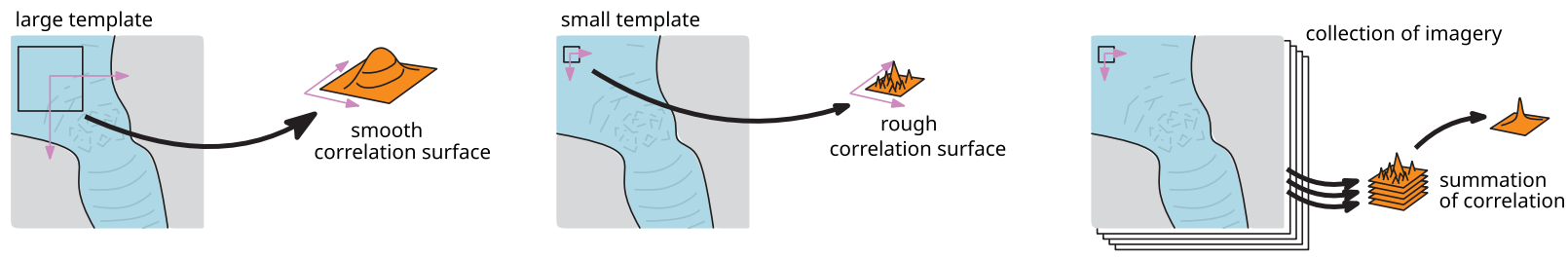

Fig. 2. Schematic illustration of the difference between regular single image pair matching and time averaged ensemble matching. Left: single image pair matching requires the application of large window sizes to achieve smooth and clearly defined correlation maxima. Middle: The application of small window sizes and single image pair matching leads to noisy correlation surfaces with small signal-to-noise ratios. Right: Within ensemble matching, the individual noisy correlation surfaces are combined (here: summed) over time to achieve clear correlation peak with high signal-to-noise ratio despite small window sizes.

stack size of several hundreds or thousands of images is included (Westerweel and others, 2004). For a highly fractured crevasse field, a smaller sample might suffice.

\section{Sub-pixel localization}

Several methods exist to estimate the localization of the correlation peak, which is inherently at pixel level, at sub-pixel resolution. A main difference between the method's implementation is the amount of information needed from the correlation scores surrounding the maximum. Either the directly neighboring pixels are used that are next to the highest score, independently estimating the peak along each axis. A second feature to consider is the shape of the peak, which can be modeled by a parabolic (Argyriou and Vlachos, 2005), Gaussian (Willert and Gharib, 1991) or triangular (Olson and Coombs, 1991) function. Other approaches use all pixels surrounding the highest correlation score, which is the case for center-of-mass, a 2D Gaussian (Nobach and Honkanen, 2005), or a spline (Fahnestock and others, 2016) function. The former methods are most popular due to their low computational power, while more computationally expensive methods like upsampling of the imagery and/or its correlation surface is also possible and can achieve precisions in the order of $1 / 20$ of a pixel (Debella-Gilo and Kääb, 2011). In this study, we investigate all the above mentioned methods against upsampling, as systematic errors are present in the different localization methods. It is therefore of importance to assess these different methods for their preferences to integer value displacements, known as peaklocking (or pixel-locking).

\section{Data}

\section{Sentinel-2 high repeat satellite imagery}

The EU/ESA Copernicus Sentinel-2 mission consists of a constellation of two medium resolution satellites recording visible to shortwave infrared imagery with a spatial resolution down to 10 meter. The first satellite of this mission, Sentinel-2A, was launched in June 2015 and has a same-orbit repeat cycle of 10 days. With the launch of Sentinel-2B in March 2017 into the same orbital plane, this revisit decreased to 5 days from the same orbit. The high dynamic range (12 bit raw) of the Sentinel-2 images makes them ideal for glacier velocity extraction as also areas with little surface contrast show often patterns useful for offset tracking. For correct displacement estimates, imagery from the same orbital track needs to be used (Kääb and others, 2016), as orthorectification errors due to erroneous or outdated elevation models distort the images. Methods are available to correct for these artifacts and make the use of imagery from alongside orbits possible (Altena and Kääb, 2017). However, in this study, such methods are not the focus and not needed, as the consistent same-orbit monitoring by Sentinel-2 provides enough imagery suited to demonstrate the method and derive a useful velocity field over the Khumbu icefall.

\section{Location-aware sport wearables}

For independent comparison to our velocity data, we use trajectory data collected by high-altitude mountaineers. These climbers move through the Khumbu icefall while equipped with location-aware wearables. These handheld GPSs have sensors that are able to record position, altitude and heading during outdoor activity. Afterwards the data can be uploaded to online sports diaries, where these can be shared either within the respective communities or archived into a private collection. Accumulation of such spatio-temporal datasets can be used to extract geographic information, mostly done through trajectory clustering, patterns or prediction (Giannotti and Pedreschi, 2008).

These types of positioning data can be used in different ways, depending on spatial and temporal selection criteria. Definitions are therefore given about such groupings for this data-type. For instance, a 'trajectory' is a collection of positioning points connected to each other by an increasing and ordered timestamp. A 'trail' is a collection of positioning points on a line without a time instance. If several trajectories are close together in space and time they are called a 'flock'. Then, when a flock is in close proximity to a trail, they are called a 'convoy'.

During a climbing ascent through the icefall, the data collection is thus as a convoy. However, in our case, the multi-temporal sequences can be seen as being within an environment that changes over time (Wiratma and others, 2017). In our situation, we assume the flock follows a trail, but the trail has a temporal character; as the glacier moves with the trail on top. Consequently, glacier velocity can be extracted over time from different flocks.

\section{Implementation}

The general workflow of our implementation is illustrated in Figure 3. The image collection is used to generate a general scene. This general appearance of the icefall is used to identify correct imagery without much cloud cover. If selected images have the correct time separation between each other, they are matched and used to build the ensemble correlation surface. The details of these steps are discussed in the sections hereafter.

\section{Cloud-free scene classification}

Cloud cover hampers the ability to extract within-season imagery for many optical Earth observation systems like Landsat (Ju and Roy, 2008). The optical instruments of Sentinel-2 are also hampered by the cloud coverage, but this constellation has an increased revisit rate of 5 days, increasing the possibility to get many pairs of images with a short acquisition interval even if a number of scenes are affected by cloud cover. Due to the resulting large data volumes, the collection of useful imagery requires automatic 


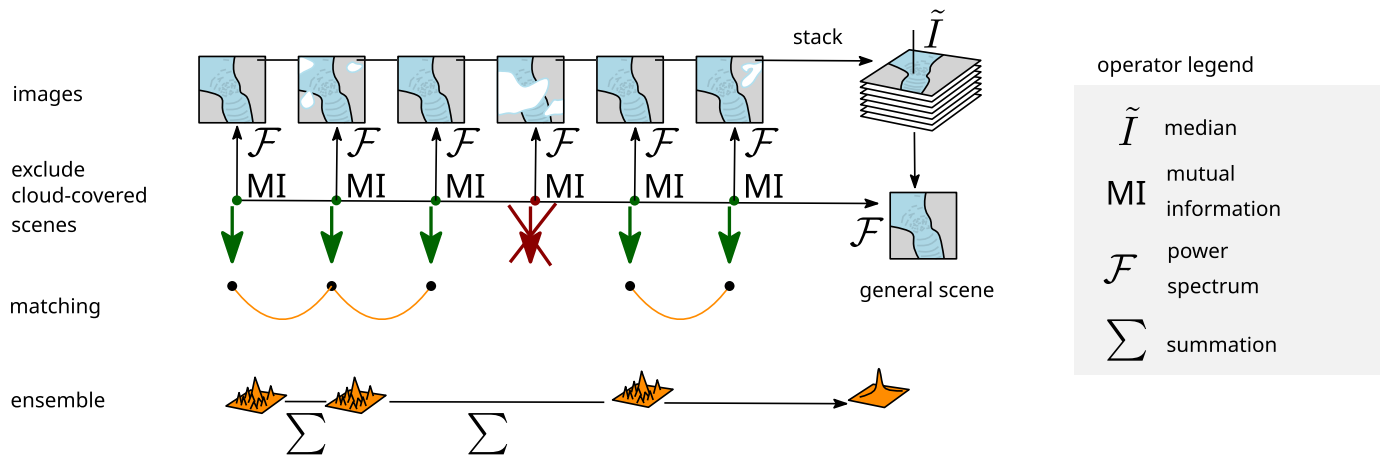

Fig. 3. Schematic illustration of the general workflow adopted in this study.

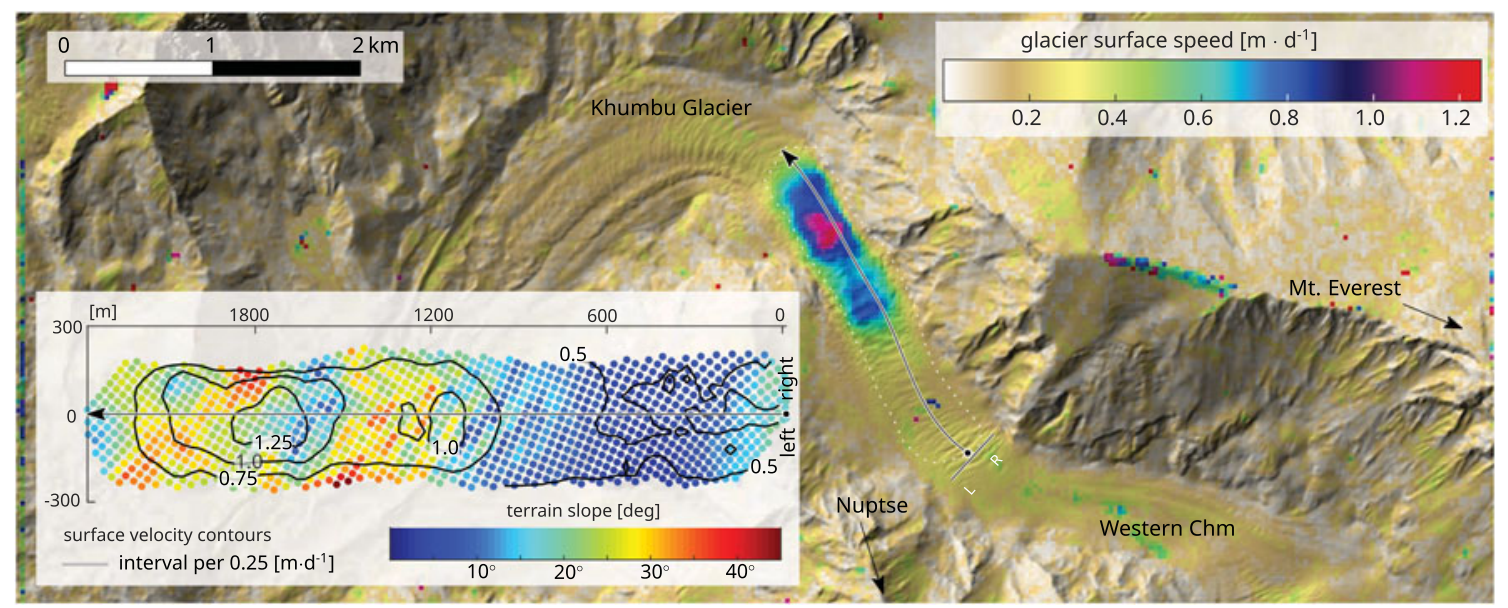

Fig. 4. Hillshading drapped with colorcoded surface velocities from ensemble matching over the Khumbu icefall, given in meter per day $\left(m d^{-1}\right)$. The template used is $30 \mathrm{~m}$ wide, sub-pixel localization is done with $2 \mathrm{D}$-spline interpolation, and peak-locking correction was applied through a secondary off-setted displacement estimation. The results from this ensemble matching can be compared to the results from standard single-pair matching in Figure 5. The inset on the left shows colorcoded slope estimates from a very high-resolution spaceborne digital elevation model (DEM) from Shean (2017) along a flowline, as indicated by the black arrow. Overlayed are the same ensemble velocity estimates as the image, but this time as contours.

classification of cloud cover. Global cloud cover products in the Sentinel-2 meta-data can be used, but these would exclude partly clouded or highly clouded scenes, even if the very location of interest (here the icefall) is actually cloudfree. The detection of clouds in high mountain terrain is challenging due to snow cover and topographic shadowing. Therefore, we use all the images and stack statistics (median) at the pixel level to generate a scene composite (or stack-median, $\tilde{\mathbf{I}}$ ) (Winsvold and others, 2016). The stack-median is then converted by a $2 \mathrm{D}$ Fourier transform, and its absolute values are taken and transformed by their natural logarithm $(\ln \|\mathcal{F}(\tilde{\mathbf{I}})\|)$. These operations result in a distinct pattern description, particularly present in natural images (Torralba and Oliva, 2003), and gives a mean to compare against other transformed images. Thus, every image is transformed by these operations, and the resulting image description is matched against the stack-median. The similarity metric calculated for this comparison is mutual information (Viola and Wells III, 1997). These mutual information scores are ultimately used to classify imagery into cloud-free and cloud-covered. This class separation is achieved through a two-class $k$-means procedure, resulting in an binary cloud-cover attribute to each image that is used to build the collection of images suitable for ensemble matching.

\section{Icefall displacement from Sentinel-2}

Currently, the absolute localization of Sentinel-2 is done through its sensor readings, hence its variability is in the order of 10 meter
(Gascon and others, 2017). Each image is therefore in our study co-registered by simple translation to the stack-median, using conventional image matching over stable terrain (Leprince and others, 2007), and each individual image is interpolated towards this base image. The stack-median is thus also affected by the same co-registration noise. However, these varying offsets do not seem to cause much blurring in the stack-median. This might however be the case for image collections with worse registration accuracy than Sentinel-2, or when significantly less images are stacked.

The individual images are then ordered into pairs from the same relative orbital track (in this case R076) and matched if they are separated by 10 days. This time-span seems to be a good compromise between significant spatial movement in relation to degradation of traceable appearance as ice flows through the icefall, where it melts and fractures. Hence, imagery of the same Sentinel-2 orbit and satellite is matched with each other. In this way also data from January 2016 onwards can be used, the time when only Sentinel-2A was in orbit. The last acquisitions used in this study are from October 2019. Given the time-span constrain of 10 days, a total of 53 pairs are matched against each other, with a template $\left(\mathbf{I}_{\mathrm{p}}\right)$ size of three pixels, while the search space is five pixels wide.

\section{Icefall displacement from GPS trajectories}

The mountain climber trajectories of an ascending (3rd of May) and a descending track (18th of May) were used for comparison 
Table 1. Group statistics of on-glacier sub-pixel localization, against up-sampled imagery

\begin{tabular}{|c|c|c|c|c|c|c|c|c|}
\hline & \multicolumn{4}{|c|}{ X-component, East-West } & \multicolumn{4}{|c|}{ Y-component, North-South } \\
\hline & Mean & Median & Std & Mad & Mean & Median & Std & Mad \\
\hline Para & -0.005 & -0.003 & 0.083 & 0.042 & -0.005 & -0.000 & 0.066 & 0.036 \\
\hline Gaussian & -0.020 & -0.014 & 0.097 & 0.056 & -0.017 & -0.004 & 0.097 & 0.063 \\
\hline Triangular & +0.093 & -0.019 & 6.369 & 0.236 & +0.132 & -0.001 & 6.740 & 0.273 \\
\hline Spline & -0.001 & -0.002 & 0.084 & 0.038 & +0.001 & +0.003 & 0.064 & 0.033 \\
\hline Center of mass & +0.007 & +0.002 & 0.078 & 0.047 & -0.001 & +0.001 & 0.072 & 0.044 \\
\hline 2D Gaussian & -0.027 & -0.020 & 1.751 & 0.119 & +0.002 & +0.000 & 0.361 & 0.119 \\
\hline
\end{tabular}

The smallest entity for each measure is highlighted.

to our surface velocity estimate. The tracks come from a Norwegian climbing team that used Garmin inReach satellite positioning devices as they climbed through Khumbu icefall in 2019, toward Camp II and Mount Everest. One of the devices has been set to record every minute, while another member recorded at a $10 \mathrm{~min}$ interval (. and + in Fig. 1, respectively). For a large extent, the path goes straight against the flow direction of the glacier. In addition, the positioning data are very noisy, most presumably due to multi-path signals from the surrounding peaks and steep ice walls (i.e. the large spread in elevation in the subplots of Fig. 1). There is only a small part of the route where the trail is perpendicular to the flow, and it is this region, which is here used as validation against the ensemble matching estimate. The trajectories were first pre-processed through a Hampel filter (Lee and Krumm, 2011) and then a cubic smoothing spline was interpolated through these points. A smoothing parameter of $5 \cdot 10^{-5}$ was chosen and checked for consistency with the $10 \mathrm{~min}$-interval dataset. At the velocity sampling distance of $30 \mathrm{~m}$, the closeness (Wiratma and others, 2017) of the ascending trajectory to the descending trajectory in the direction of glacier flow was then measured to extract a glacier velocity estimate.

\section{Results and discussion}

The automatic cloud detection through a stack-median and spectral matching through mutual information gives a success rate of roughly $80 \%$. While more advanced methods might work, this score is reasonable for this application, as cloud scenes might only lower the signal-to-noise, but will not introduce a velocity bias. We think the high success rate of the classification is mainly due to the large volume of Sentinel-2 imagery available in the archive that seems sufficient to generate a good composite of the general appearance of the scence by the stack-median. Further success is due to the frequency distribution of clouds; their signature in the power spectrum seems to be sufficiently different from topography. A seasonal dependency is present in our classification, as many scenes within the summer season are assigned as cloudy imagery. As the selected scenes fall outside of the monsoon season, the extracted velocities from the ensemble matching will mostly represent the winter velocities on Khumbu Glacier.

\section{Icefall velocity field}

The absolute surface velocity estimation over Khumbu icefall is illustrated in Figure 4 . Where the maximum velocity seems to be just over $1 \mathrm{~m} \mathrm{~d}^{-1}$. Two distinct patches of fast flow occur after each other, aligning with the steepest sections of the icefall. It seems these faster velocities are situated just upstream of an increase in slope (see inset of Fig. 4). These spatial patterns of velocity differences show the achievable detail of this method. Though we should note that the velocity shown here are planimetric velocity and these estimates are not corrected or normalized with the slope of the terrain.

Figure 5 shows three examples of pair-wise template matching for May 2018 with Sentinel-2 data. The upperleft frame in Figure 5 shows an estimate of the annual velocity from ITSLIVE for 2018, generated using auto-RIFT (Gardner and others, 2018). This estimate is based on Landsat 8 imagery, has a sampling interval of $240 \mathrm{~m}$, and uses median statistics over a large collection of velocity fields. These annual estimates clearly show that a long separation time up to hundreds of days complicates the generation of sufficient results over the icefall, though the slower moving ice at the tongue is captured. The other insets are velocity estimates stemming from two Sentinel-2B scenes (on 8 and 18 May 2018), which is in the same time period as the GPS trajectories, as shown in Figure 1. The different solutions shown in Figure 5 are due to the use of different template sizes, but the sampling distance is every $30 \mathrm{~m}$. Foremostly, the smoothing and thus dampening of the signal is apparent, as the template size increases. The amount of spatial detail present in the $30 \mathrm{~m}$ solution is comparable to the ensemble matching, it is though significantly more affected by noise. There was no filtering applied to the ensemble solution (Fig. 4), and there seems no filtering necessary which is a great benefit. The velocities in early May 2018

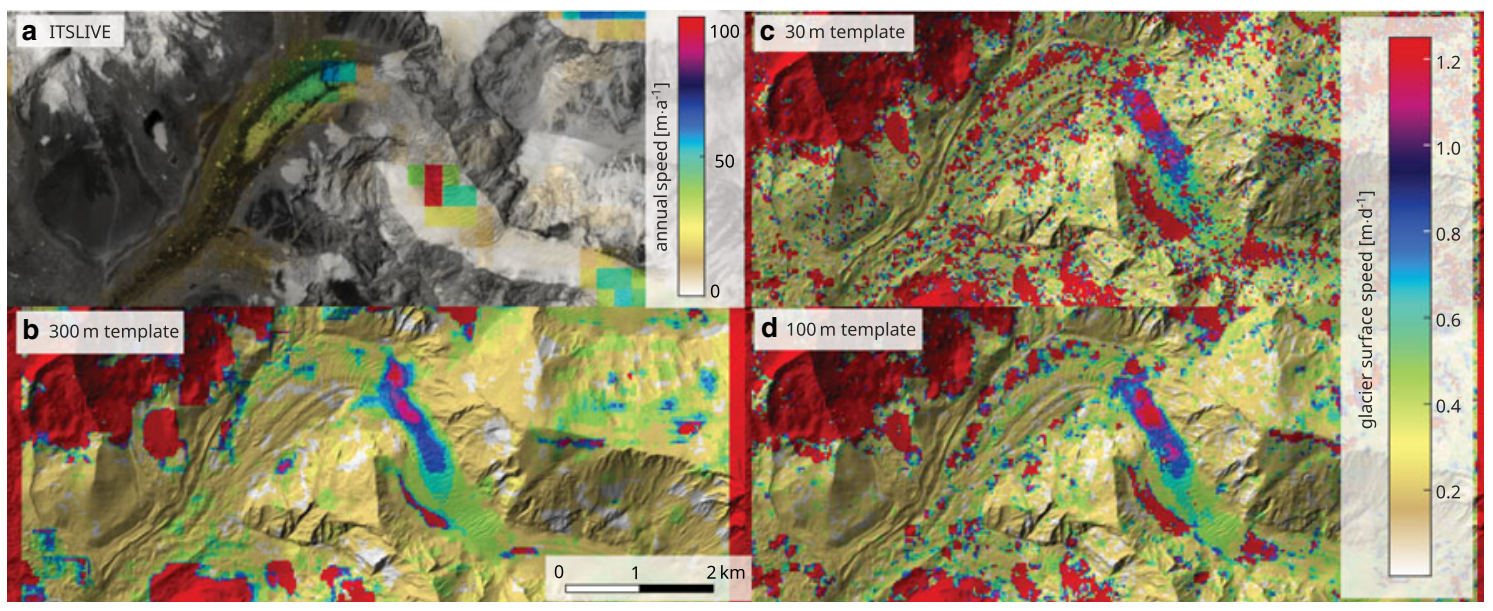

Fig. 5. (a) Annual glacier velocity estimate of 2018 over Khumbu Glacier from ITSLIVE. These velocity estimates are low and are given a different colorcoding than in the other panels. The background is a Sentinel-2B scene on 8 May 2018, which is one of the images used for velocity extraction of the other velocity estimates in this figure. (b-d) 'Classical' velocity estimate from a pair of Sentinel-2 images with 10 day interval, but different sizes of template windows used. 
Fig. 6. Histograms of shift differences between several subpixel estimators and the reference dataset are shown in (a) and (b), the reference dataset was upsampled by a factor of ten. The distributions are a selection of all the pixels, these are all estimates that shifted by $1 / 10$ of a pixel according to the reference dataset. (a) Distribution of -0.1 pixel displacement, (b) distribution of +0.1 pixel displacement. (subpixel estimator - upsampled imagery) 0.06 moved -0.1 pixel in reference imagery

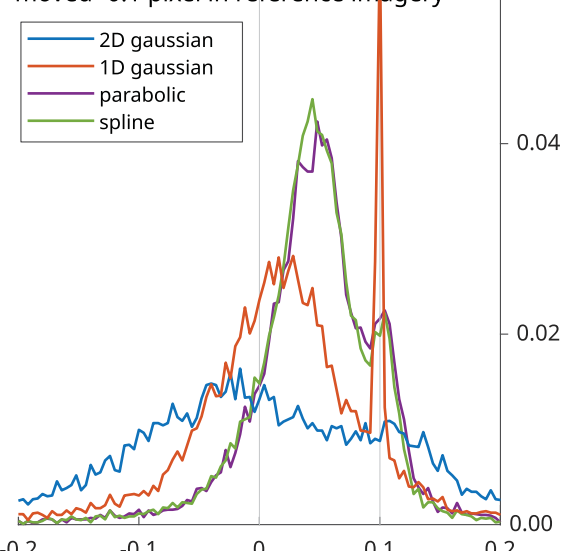

b

(subpixel estimator - upsampled imagery) $\leftarrow 0.06$ moved +0.1 pixel in reference imagery

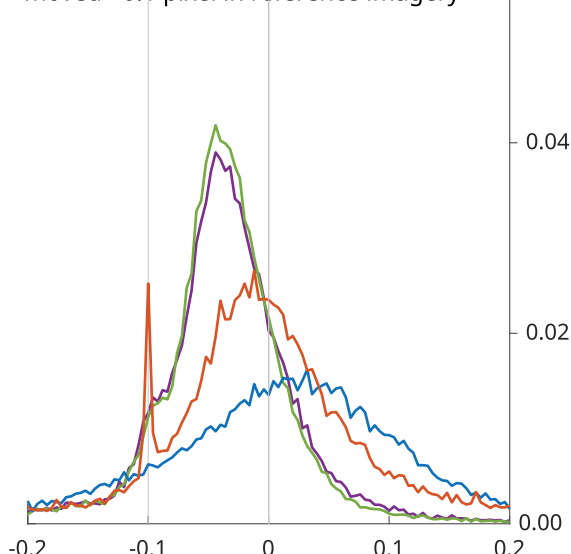

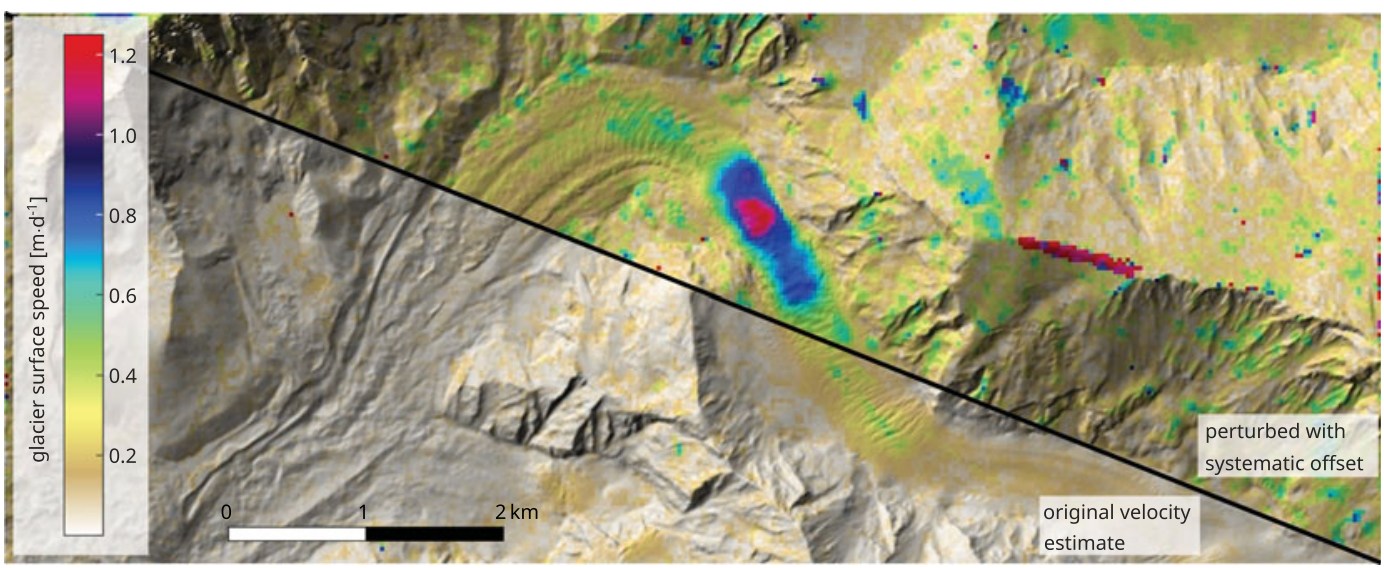

Fig. 7. Split window between velocity estimates for ensemble matching with original co-registered imagery, and ensemble matching with a systematic offset of half a pixel, which is later removed. Both sub-pixel localization estimates use the spline interpolation. The mean velocity of these estimates are shown in Figure 4.

Fig. 8. Histograms of shift differences between severa subpixel estimators with a compensation by a systematic offset of half a pixel and the reference dataset are shown in (a) and (b), the reference dataset was upsampled by a factor of ten. Here, the distribution is shown for all pixels which are shifted by $1 / 10$ of a pixel according to the reference dataset. (a) Distribution of $-1 / 10$ pixel displacement, (b) distribu tion of $+1 / 10$ pixel displacement. (subpixel estimator - scaled imagery) moved -0.1 pixel in scaled imagery

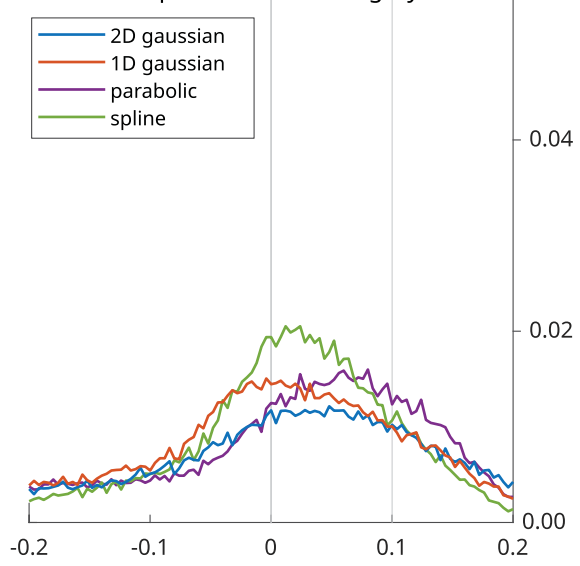

(subpixel estimator - scaled imagery)

0.06 moved +0.1 pixel in scaled imagery

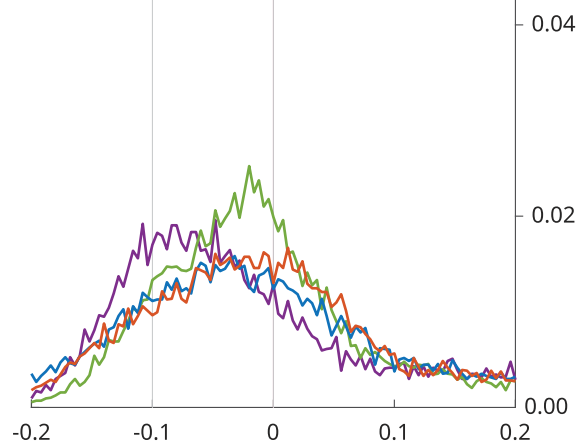

seem to be faster than the ensemble solution, which uses pairs mostly outside of the spring and summer season.

\section{Sub-pixel localization}

In order to assess the different sub-pixel localization approaches, we up-sampled all imagery by a factor of ten, to get an estimate to be used as reference. The statistics of their comparison against this dataset is listed in Table 1 Both classical and robust statistical measures are shown, as especially the triangular method has many outliers within. Representing the correlation peak through a triangular shape assumes an ideal measurement system, where simple translation resolves in an ideal pulse in the correlation surface. However, due to flow variation of the ice and change in 

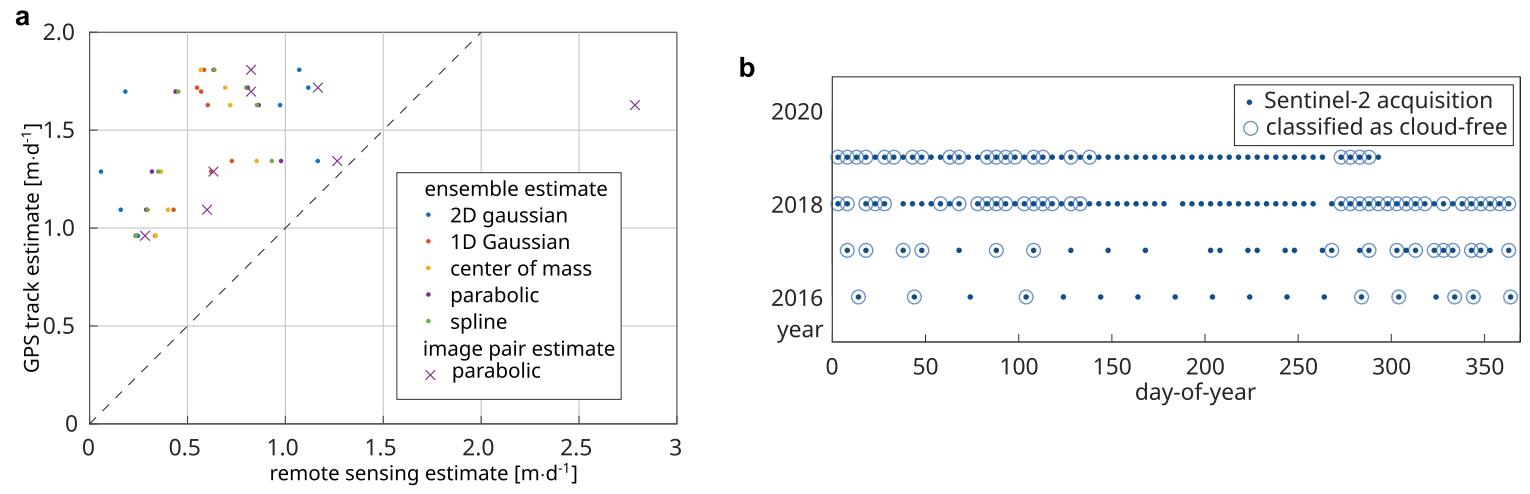

Fig. 9. (a) Comparison of the different subpixel estimates to the GPS displacements, indicated by circles $(\bullet)$. The crosses $(x)$ stem from a single pair of Sentinel-2 images in spring, this velocity field is also shown in Figure 5c. (b) Shows the image time serie of Sentinel-2 and the associated cloud-free classification. The monsoon seems to create a gap in the summer months. (a) GPS comparison to ensemble, (b) Sentinel-2 scene selection over time

appearance of the terrain, smoothing of the top of the correlation peak occurs. Neighboring correlation scores can therefore be relatively high, deviating from the pointy model of a triangle and causing location estimates far larger than half a pixel. Thus, from here onwards the triangular localization is neglected from our analysis.

The statistics in Table 1 give a general overview of the localization performance, but also an optimistic view of precision. Since large portions of the glacier are flowing slowly, these regions are overrepresented in these statistics. In general, this might not be an issue, but pixel-localization methods suffer from 'peaklocking', where a preference exist toward integer values (Willert and Gharib, 1991; Westerweel, 1997) causing a bias. This bias stems from large variations of correlation values around the correlation peak, hence the localization methods will prefer to stay close to integer value. This large variation in correlation scores is especially the case for ensemble matching, where correlation surfaces with low signal-to-noise ratios are summed-up.

The reference dataset used for this assessment is without subpixel localization, and has thus a resolution of 0.1 pixel. Hence, grouping the statistics into these increments gives an improved insight into the peak-locking bias present in the estimates, as it gives the opportunity to distangle the group statistics into subdistributions. Such distributions are shown in Figures $6 a$ and b for on-glacier velocity estimates which have a displacement of one-tenth of a pixel in the reference dataset. Here the differences between the reference dataset and the different peak localization methods are shown. If no-bias would exist then the mode and mean would be centered around zero. However, there is a significant skew present, especially for the parabolic and spline localization. Furthermore, a secondary peak is present at a tenth of a pixel distance (in line with the vertical lightgray axis). This peak is caused because the localization method was not able to estimate within bounds of the peak, being further than half a pixel. The tendency in both sub-distributions is toward this secondary peak, showing the sensitivity for peak-locking. From these statistics, the Gaussian methods seem to have less dependency on peak-locking (Westerweel, 1997). However, for the onedimensional Gaussian estimator, a significant amount of peak locations could not be estimated. This can stem from the natural logarithm function within the Gaussian estimator that transforms white noise asymmetrically (Anthony and Granick, 2009).

Compensation for peak-locking can be done by re-interpolation, looking at the group statistics and smooth accordingly (Christensen, 2004). However, the distribution of velocities needs to stretch over multiple integer values, which is not the case here. Another option is to systematically offset one of the images by half a pixel (Shimizu and Okutomi, 2002). The introduced offset is also affected by peak-locking, but its dependency is in the other direction and can thus compensate for the peaklocking bias. This method is applied here, and the two results for spline interpolation are shown in the Figure 7. Variation on stable terrain is considerably more in the offset version in respect to the original. When combined, an estimate with less bias can be calculated, as shown in Figure 4. A similar analysis as is done previously with the standard estimation can be done with the adjusted estimates, as is shown in Figures $8 \mathrm{a}$ and b. Here a clear difference seems to emerge between the parabolic and the spline estimators. The parabolic estimate still has large peaklocking effects, while the spline method seems to align most centered to zero, with less skew than the others. While the Gaussian methods have a slight peak-locking dependency and more variability. The one-dimensional Gaussian method has a considerable amount of location estimates outside of the sub-pixel range (Fig. 6), and for these estimates, it is not possible to be compensated. Hence, here as well, the two-dimensional method is preferred over the one-dimensional.

\section{GPS trajectories through the icefall}

There is a general and consistent underestimation of the ensemble matching estimates in relation to the in situ measurements (Fig. 9a). This can be due to (i); the noisy nature of satellite positioning; or (ii) the measurement interval of both measurements (Fig. 9b), as the GPS velocities are in spring and might not be in line with annual velocities. Thus, the short time interval of the GPS velocities might capture higher spring velocities that stem from a larger sliding component underneath the icefall than the average during the entire melting period. This is supported by the individual matching estimates in summer, as shown in Figure 5, which give also higher estimates than the ensemble estimate. This is also observable in Figure 9a, where individual velocity estimates $(x)$ are consistently larger than the ensemble estimate $(\bullet)$.

\section{Conclusions and perspective}

To the best of our knowledge, our study is the first to test ensemble matching outside of controlled laboratory environments and in a typical remote-sensing setting. Eventhough it is a specialized technique, it has an application domain in combination with the highrepeat imagery of Sentinel-2. Previously, it has not been possible or at least difficult to extract velocity estimates over fast-flowing and fast-changing glacier zones such as icefalls, as demonstrated here over Khumbu icefall (see figures in Scherler and others, 2008; Rowan and others, 2015; Haritashya and others, 2015; Rounce and others, 2018). Typically, the velocities on the tongue of a glacier are measured using repeat optical satellite images, as in the 
case of Khumbu Glacier is downstream from Everest base camp (Bolch and others, 2008). The constrain set by large window sizes in classical pattern matching is not limited to optical remote sensing alone, and SAR speckle tracking (Quincey and others, 2009) has not been able to resolve this part of Khumbu Glacier either. However, the ensemble matching technique is transferable to the SAR domain, and we think is able to enhance SAR offset tracking results in a similar fashion as presented here.

Recently, encouraging results have been produced through the use of stacking of displacement fields and using weighting or median filtering, generating products with a resolution at $50 \mathrm{~m}$ (Nobakht and others, 2014; Millan and others, 2019) with Sentinel-2, or 100/125 $\mathrm{m}$ with Landsat 8 (Shen and others, 2018; Gardner and others, 2018). However, these results are based on large window sizes followed by spatial smoothing, and the such-generated estimates might thus not align with the true velocity gradients. Such smoothing is not done in this study, and the inherent detail and errors of the icefall surface velocity are clearly observed. Neither did we apply any postprocessing in this study, while except from steep mountain ridges, clear outliers do not seem to occur on the icefall in our estimate. This makes the matching more transparent, straight forward and complete than for many previous approaches where method differences lie often more in the postprocessing than in the matching itself (Heid and Kääb, 2012). The amount of imagery used here (53 pairs) might by far be sufficient, which shows the opportunity to use this technique to resolve annual or even seasonal estimates with less pairs. In addition, we identify systematic differences between the different sub-pixel localization methods. From our analysis, we can state that there seems to be a superiority in spline methods. However, compensation for peak-locking is needed. Practically, this means the ensemble matching needs to run a second time. Because the correlation score surface in the spatial domain is used, other sub-pixel localization methods that are based on the frequency domain are not assessed here (i.e.: TPSS Leprince and others, 2007, sinc-function Rösgen, 2003). Because of the summation or stacking of correlation surfaces, it is neither possible to assess iterative sub-pixel estimators that work on the imagery directly (i.e. differential methods Lucas and Kanade, 1981). These methods might be less affected by peak-locking, thus our results can only for a limited amount be transferred to image pair matching.

Lastly, the limited amount of in situ GPS trajectory data restricts our comparison effort, but we demonstrate this data type as an interesting source to extract information in unconventional ways.

Acknowledgements. We thank Didrik Bakke Dukefoss and Thomas Erling Lone, the high-altitude mountaineers that were willing to share their positioning data of the tracks which they climbed through Khumbu icefall while making a successful ascent to Mount Everest. We also thank two anonymous reviewers for their input, which helped to improve the manuscript, and Rachel Carr for handling the review process as editor. This research and development has been conducted through support from the European Union FP7 ERC project ICEMASS (320816) and through ESA Living Planet Fellowship to B.A., Glaciers-CCI, CCI+ and EE10 HARMONY projects (4000125560/18/ I-NS, 4000109873/14/I-NB, 4000127593/19/I/NB, 4000127656/19/NL/FF/gp). The code will be available on http://www.runmycode.org/coder/view/3479.

Supplementary material. To view supplementary material for this article, please visit https://doi.org/10.1017/jog.2020.66

\section{References}

Altena B and Kääb A (2017) Elevation change and improved velocity retrieval using orthorectified optical satellite data from different orbits. Remote Sensing 9(3), 300. doi: 10.3390/rs9030300.
Anthony S and Granick S (2009) Image analysis with rapid and accurate twodimensional Gaussian fitting. Langmuir 25(14), 8152-8160. doi: 10.1021/ la900393v.

Argyriou V and Vlachos T (2005) Performance study of gradient correlation for sub-pixel motion estimation in the frequency domain. IEEE Proceedings-Vision, Image and Signal Processing 152(1), 107-114. doi: 10. 1049/ip-vis:20051073.

Armstrong WH, Anderson RS and Fahnestock MA (2017) Spatial patterns of summer speedup on South central Alaska glaciers. Geophysical Research Letters 44, 1-10. doi: 10.1002/2017GL074370.

Bolch T, Buchroithner M, Peters J, Baessler M and Bajracharya S (2008) Identification of glacier motion and potentially dangerous glacial lakes in the Mt. Everest region/Nepal using spaceborne imagery. Natural Hazards and Earth System Sciences 8(6), 1329-1340. doi: 10.5194/nhess-8-1329-2008.

Christensen K (2004) The influence of peak-locking errors on turbulence statistics computed from PIV ensembles. Experiments in Fluids 36(3), 484-497. doi: 10.1007/s00348-003-0754-2.

Colgan W and 6 others (2016) Glacier crevasses: observations, models, and mass balance implications. Reviews of Geophysics 54(1), 119-161. doi: 10. 1002/2015RG000504.

Debella-Gilo M and Kääb A (2011) Sub-pixel precision image matching for measuring surface displacements on mass movements using normalized cross-correlation. Remote Sensing of Environment 115(1), 130-142. doi: 10.1016/j.rse.2010.08.012.

Debella-Gilo M and Kääb A (2012) Locally adaptive template sizes for matching repeat images of Earth surface mass movements. ISPRS journal of photogrammetry and remote sensing 69, 10-28. doi: 10.1016/j.isprsjprs.2012.02.002.

Dehecq A, Gourmelen N and Trouvé E (2015) Deriving large-scale glacier velocities from a complete satellite archive: application to the PamirKarakoram-Himalaya. Remote Sensing of Environment 162, 55-66. doi: 10.1016/j.rse.2015.01.031.

Delnoij E, Westerweel J, Deen N, Kuipers J and van Swaaij W (1999) Ensemble correlation piv applied to bubble plumes rising in a bubble column. Chemical Engineering Science 54(21), 5159-5171.

Durkin W, Bartholomaus T, Willis M and Pritchard M (2017) Dynamic changes at Yahtse glacier, the most rapidly advancing tidewater glacier in Alaska. Frontiers in Earth Science 5, 21. doi: 10.3389/feart.2017.00021.

Fahnestock $\mathbf{M}$ and 5 others (2016) Rapid large-area mapping of ice flow using Landsat 8. Remote Sensing of Environment 185, 84-94. doi: 10.1016/j.rse. 2015.11.023.

Firth P and 8 others (2008) Mortality on Mount Everest, 1921-2006: descriptive study. British Medical Journal 337, a2654. doi: 10.1136/bmj.a2654.

Gardner A and 6 others (2018) Increased West Antarctic and unchanged East Antarctic ice discharge over the last 7 years. The Cryosphere 12(2), 521-547. doi: 10.5194/tc-12-521-2018.

Gascon F and 22 others (2017) Copernicus Sentinel-2A calibration and products validation status. Remote Sensing 9(6), 584. doi: 10.3390/rs9060584.

Giannotti F and Pedreschi D (2008) Mobility, data mining and privacy: geographic knowledge discovery, 1st edn. Heidelberg: Springer Science \& Business Media. doi: 10.1007/978-3-540-75177-9.

Gilbert A and 6 others (2020) The influence of water percolation through crevasses on the thermal regime of Himalayan mountain glaciers. The Cryosphere 14, 1273-1288. doi: 10.5194/tc-14-1273-2020.

Giordan D and 5 others (2016) A low-cost optical remote sensing application for glacier deformation monitoring in an alpine environment. Sensors 16 (10), 1750. doi: 10.3390/s16101750.

Haritashya U, Pleasants M and Copland L (2015) Assessment of the evolution in velocity of two debris-covered valley glaciers in Nepal and New Zealand. Geografiska Annaler: Series A, Physical Geography 97(4), 737751. doi: 10.1111 /geoa.12112.

Harper J and Humphrey N (1995) Borehole video analysis of a temperate glacier's englacial and subglacial structure: implications for glacier flow models. Geology 23(10), 901-904.

Heid T and Kääb A (2012) Repeat optical satellite images reveal widespread and long term decrease in land-terminating glacier speeds. The Cryosphere 6(2), 467-478. doi: 10.5194/tc-6-467-2012.

Jarvis G and Clarke G (1974) Thermal effects of crevassing on Steele glacier, Yukon territory, Canada. Journal of Glaciology 13(68), 243-254. doi: 10. 3189/S0022143000023054.

Ju J and Roy D (2008) The availability of cloud-free Landsat ETM+ data over the conterminous United States and globally. Remote Sensing of Environment 112(3), 1196-1211. doi: 10.1016/j.rse.2007.08.011. 
Kääb A and 5 others (2016) Glacier remote sensing using Sentinel-2. Part I: radiometric and geometric performance, and application to ice velocity. Remote sensing 8(7), 2072-4292. doi: 10.3390/rs8070598.

Kääb A and Vollmer M (2000) Surface geometry, thickness changes and flow fields on creeping mountain permafrost: automatic extraction by digital image analysis. Permafrost and Periglacial Processes 11(4), 315-326.

Kodama H and Mae S (1976) The flow of glaciers in the Khumbu region. Journal of the Japanese Society of Snow and Ice 38, 31-36.

Lee WC and Krumm J (2011) Computing with spatial trajectories, chapter Trajectory preprocessing. New York: Springer, pp. 3-33. doi: 10.1007/978 1-4614-1629-6.

Leprince S, Barbot S, Ayoub F and Avouac JP (2007) Automatic and precise orthorectification, coregistration, and subpixel correlation of satellite images, application to ground deformation measurements. IEEE Transactions on Geoscience and Remote Sensing 45(6), 1529-1558. doi: 10.1109/TGRS.2006. 888937.

Lucas B and Kanade T (1981) An iterative image registration technique with an application to stereo vision. Proceedings of the 7 th international conference on artificial intelligence (IJCAI), Vancouver, British Columbia, pp. 674-679.

Meinhart C, Wereley S and Santiago J (2000) A PIV algorithm for estimating time-averaged velocity fields. Journal of Fluids Engineering 122(2), 285-289. doi: $10.1115 / 1.483256$.

Messerli A and Grinsted A (2015) Image georectification and feature tracking toolbox: ImGRAFT. Geoscientific Instrumentation, Methods and Data Systems 4(1), 23-34. doi: 10.5194/gi-4-23-2015.

Millan R and 6 others (2019) Mapping surface flow velocity of glaciers at regional scale using a multiple sensors approach. Remote Sensing 11(21), 2498. doi: $10.3390 / \mathrm{rs} 11212498$.

Moore G, Cristofanelli P, Bonasoni P, Verza G and Semple J (2017) Automatic weather station observations of the April 2014 Mount Everest avalanche. Arctic, Antarctic, and Alpine Research 49(2), 321-330. doi: 10 1657/AAAR0016-059.

Nobach H and Honkanen M (2005) Two-dimensional Gaussian regression for sub-pixel displacement estimation in particle image velocimetry or particle position estimation in particle tracking velocimetry. Experiments in fluids 38(4), 511-515. doi: 10.1007/s00348-005-0942-3.

Nobakht M, Motagh M, Wetzel HU, Roessner S and Kaufmann H (2014) The Inylchek glacier in Kyrgyzstan, central Asia: insight on surface kinematics from optical remote sensing imagery. Remote Sensing 6(1), 841856. doi: 10.3390/rs6010841.

Olson T and Coombs D (1991) Real-time vergence control for binocular robots. International Journal of Computer Vision 7(1), 67-89. doi: 10. 1007/BF00130490.

Quincey D, Luckman A and Benn D (2009) Quantification of Everest region glacier velocities between 1992 and 2002, using satellite radar interferometry and feature tracking. Journal of Glaciology 55(192), 596-606. doi: 10.3189/ 002214309789470987.

Rösgen T (2003) Optimal subpixel interpolation in particle image velocimetry. Experiments in Fluids 35(3), 252-256. doi: 10.1007/s00348-003-0627-8.

Rounce D, King O, McCarthy M, Shean D and Salerno F (2018) Quantifying debris thickness of debris-covered glaciers in the Everest region of Nepal through inversion of a subdebris melt model. Journal of Geophysical Research: Earth Surface 123(5), 1094-1115. doi: 10.1029/2017JF004395.

Rowan A, Egholm D, Quincey D and Glasser N (2015) Modelling the feedbacks between mass balance, ice flow and debris transport to predict the response to climate change of debris-covered glaciers in the Himalaya Earth and Planetary Science Letters 430, 427-438. doi: 10.1016/j.epsl 2015.09.004.

Scambos T, Dutkiewicz M, Wilson J and Bindschadler R (1992) Application of image cross-correlation to the measurement of glacier velocity using satellite image data. Remote Sensing of Environment 42(3), 177-186. doi: 10. 1016/0034-4257(92)90101-O.

Scherler D, Leprince S and Strecker M (2008) Glacier-surface velocities in alpine terrain from optical satellite imagery-accuracy improvement and quality assessment. Remote Sensing of Environment 112(10), 3806-3819. doi: $10.1016 /$ j.rse.2008.05.018.

Shean D (2017) High Mountain Asia 8-meter DEM mosaics derived from optical imagery, version 1. NASA National Snow and Ice Data Center Distributed Active Archive Center. doi 10.5067/KXOVQ9L172S2.

Shen Q and 5 others (2018) Recent high-resolution Antarctic ice velocity maps reveal increased mass loss in Wilkes Land, East Antarctica. Scientific reports 8(1), 4477. doi: 10.1038/s41598-018-22765-0.

Shimizu M and Okutomi M (2002) Precise subpixel estimation on area-based matching. Systems and Computers in Japan 33(7), 1-10. doi: 10.1002/scj.10098.

Stokes S, Koirala P, Wallace S and Bhandari S (2015) Tragedy on Everest: the Khumbu icefall. Emergency Medicine Journal 32(5), 418-420. doi: 10.1136/ emermed-2014-204489.

Strozzi T, Luckman A, Murray T, Wegmüller U and Werner C (2002) Glacier motion estimation using SAR offset-tracking procedures. IEEE Transactions on Geoscience and Remote Sensing 40(11), 2384-2391. doi: 10.1109/TGRS.2002.805079.

Torralba A and Oliva A (2003) Statistics of natural image categories. Network: computation in neural systems 14(3), 391-412. doi: 10.1088/0954-898X_14_3_302.

Viola P and Wells III W (1997) Alignment by maximization of mutual information. International Journal of Computer Vision 24(2), 137-154. doi: 10. 1023/A:1007958904918.

Westerweel J (1997) Fundamentals of digital particle image velocimetry. Measurement Science and Technology 8(12), 1379. doi: 10.1088/ 0957-0233/8/12/002

Westerweel J, Geelhoed P and Lindken R (2004) Single-pixel resolution ensemble correlation for micro-PIV applications. Experiments in Fluids 37(3), 375-384. doi: 10.1007/s00348-004-0826-y.

Willert C and Gharib M (1991) Digital particle image velocimetry. Experiments in fluids 10(4), 181-193. doi: 10.1007/BF00190388.

Winsvold S, Kääb A and Nuth C (2016) Regional glacier mapping using optical satellite data time series. IEEE Journal of Selected Topics in Applied Earth Observations and Remote Sensing 9(8), 3698-3711. doi: 10. 1109/JSTARS.2016.2527063.

Wiratma L, van Kreveld M and Löffler M (2017) Societal geo-innovation, chapter On measures for groups of trajectories. Lecture Notes in Geoinformation and Cartography. Cham: Springer, pp. 311-330. doi: 10. 1007/978-3-319-56759-4 


\section{Appendix}

Sub-pixel estimates of both the North-South (Fig. 10) and East-West (Fig. 11) direction here in the Appendix. The corresponding statistics and distribution plots are shown in Table 1, Figure 6 and the main text.

a

b

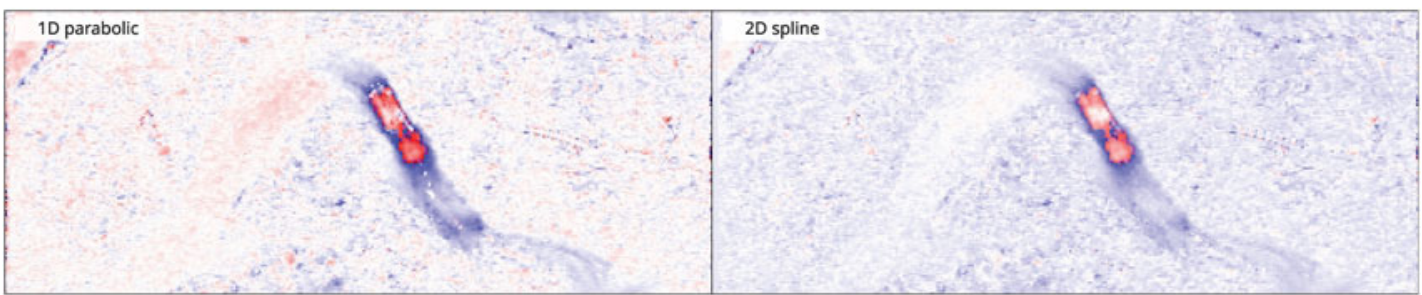

c

d

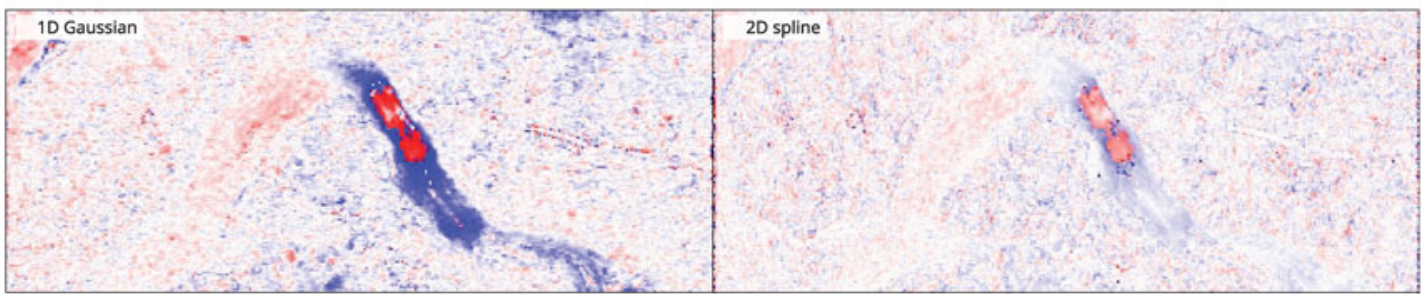

e $\quad \mathbf{f}$

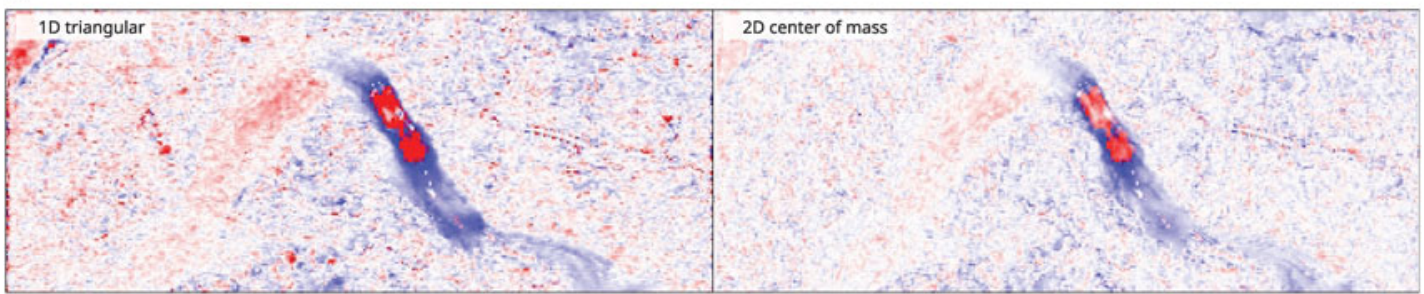

g

h

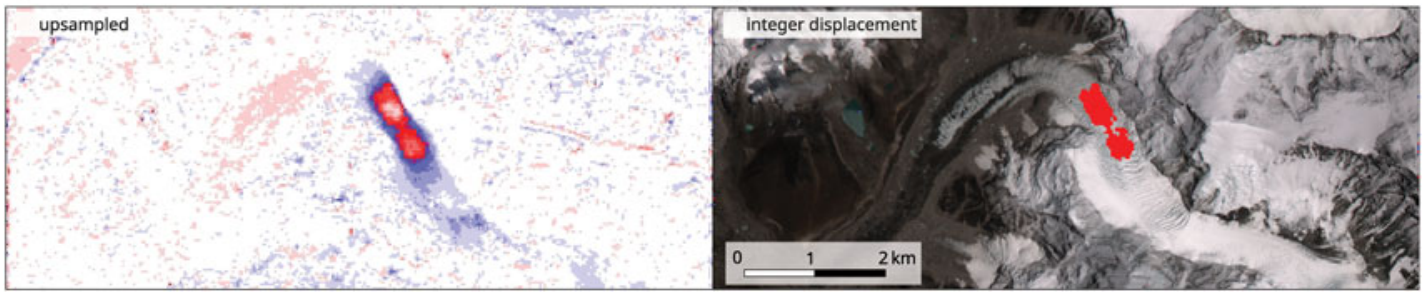

Fig. 10. Sub-pixel localization for the Y-component (North-South) of Khumbu icefall, where the color scale goes from red for -0.5 , white is 0 and blue is +0.5 , in pixel spacing. (a) 1D Parabolic; (b) 2D spline; (c) 1D Gaussian; (d) 2D Gaussian; (e) 1D triangular (f) 2D center of mass; (g) upsampled; (h) integer displacement 
a

b

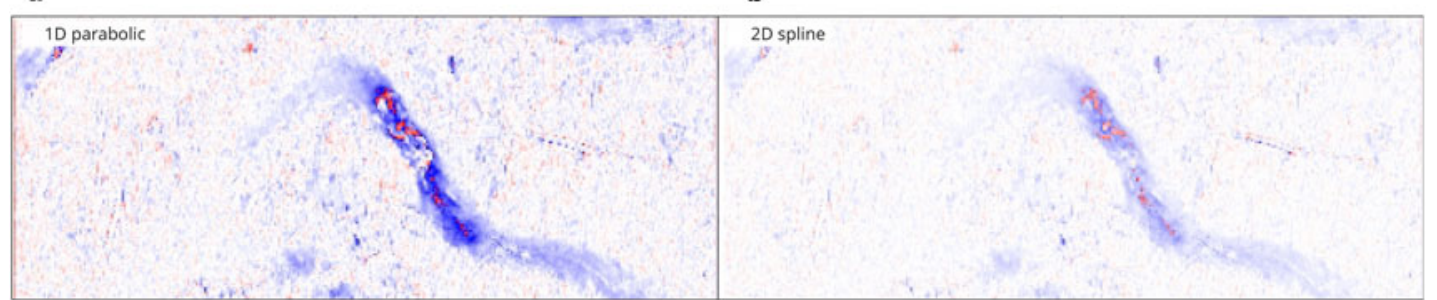

d
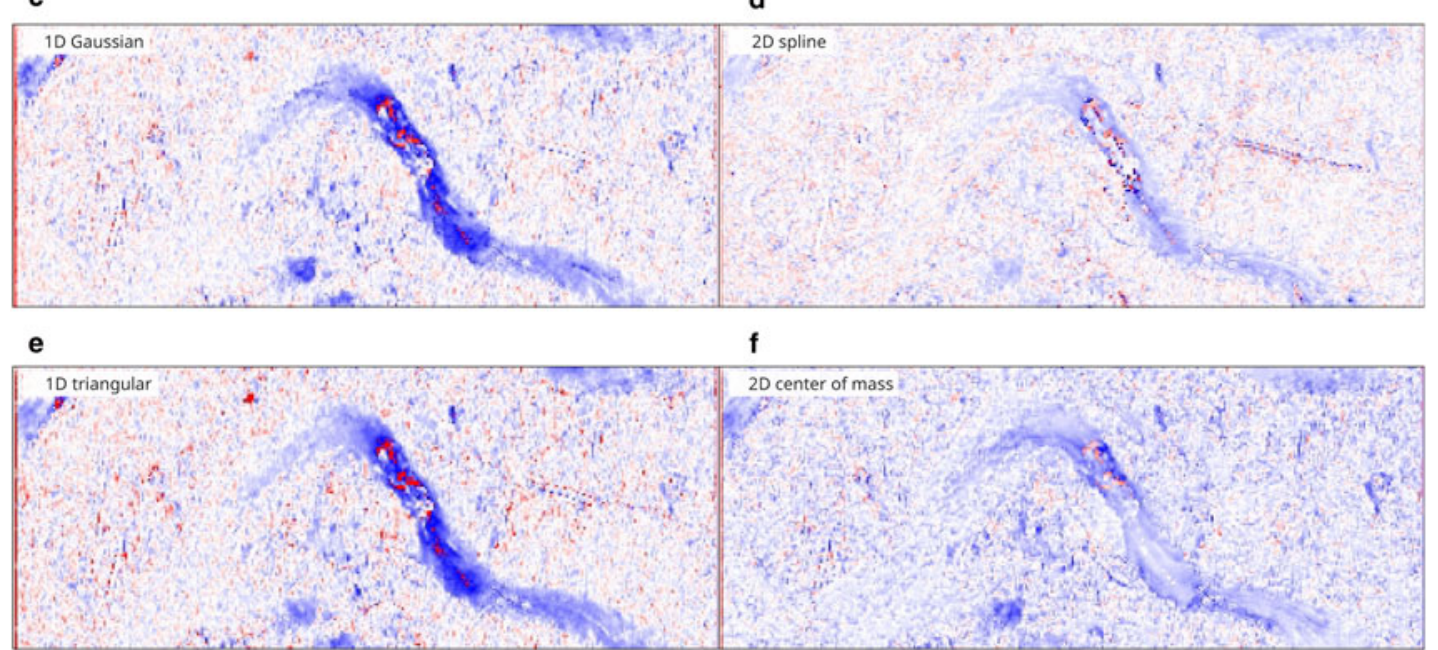

g

h

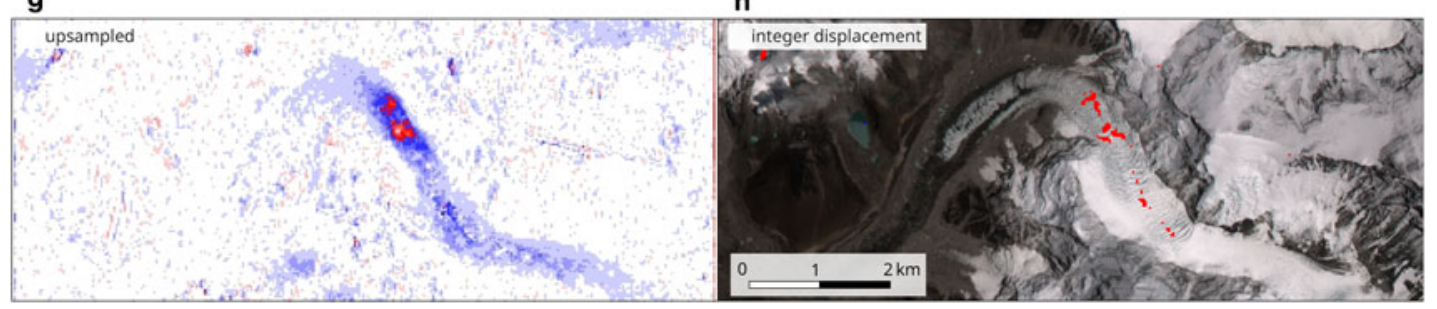

Fig. 11. Sub-pixel localization for the X-component (East-West) of Khumbu icefall, where the color scale goes from red for +0.5 , white is 0 and blue is -0.5 , in pixel spacing. (a) 1D Parabolic, (b) 2D spline, (c) 1D Gaussian, (d) 2D Gaussian, (e) 1D triangular, (f) 2D center of mass, (g) upsampled, (h) integer displacement. 